\title{
Diagnóstico Clínico de la Hematuria Vesical Enzoótica Bovina por Urianálisis de la Provincia de Oxapampa, Perú
}

\author{
Clinical Diagnostic of Bovine Enzootic Vesical Hematuria by Urinalysis in \\ Oxapampa, Peru
}

\author{
Gemma Verde Z. ${ }^{1,3}$, Mario García P. ${ }^{1}$, Alfonso Chavera C. ${ }^{2}$, Christian Gonzáles E. ${ }^{2}$, \\ Néstor Falcón P. ${ }^{1}$
}

\section{Resumen}

\begin{abstract}
El estudio se desarrolló en la provincia de Oxapampa, Región Pasco, Perú, con el objetivo de diagnosticar clínicamente la hematuria vesical enzoótica bovina (HVEB) mediante el urianálisis, acompañado de la evaluación clínica y semiológica del animal y de la inspección general del establecimiento ganadero. Se obtuvieron muestras de orina por estimulación manual perivulvar y perineal de 211 bovinos hembras, mayores de 6 meses, expuestos naturalmente al consumo del helecho común o Pteridium aquilinum $(\mathrm{Pa})$. Las muestras recolectadas fueron sometidas a una prueba tamiz con tiras reactivas (TR), evaluación físico-química (color, turbidez, peso específico y $\mathrm{pH}$ ), pruebas complementarias químicas (proteína, glucosa y bilirrubina), así como una evaluación microscópica del sedimento (EMS), prueba confirmatoria de hematuria, relevante para los casos subclínicos (microhematuria). Mediante la EMS, los casos de hematuria presentaron variadas intensidades, por lo cual se planteó una clasificación de grados de acuerdo a ellas, basado en el número de eritrocitos por campo hallados en cada muestra. Se discute la relación entre los resultados de la EMS con las TR, pruebas físico-químicas y químicas complementarias. Se encontró una prevalencia de $26.1 \%$ para la zona y los resultados del análisis estadístico indican que la edad y el intervalo al último parto pueden ser considerados como factores predisponentes de la HVEB.
\end{abstract}

Palabras clave: Hematuria Vesical Enzoótica Bovina; urianálisis; EMS; TR; Pteridium aquilinum

\footnotetext{
${ }^{1}$ Facultad de Medicina Veterinaria y Zootecnia, Universidad Peruana Cayetano Heredia, Lima, Perú

${ }^{2}$ Facultad de Medicina Veterinaria, Universidad Nacional Mayor de San Marcos, Lima, Perú

${ }^{3}$ E-mail: gemma.verde.z@upch.pe
}

Recibido: 12 de abril de 2016

Aceptado para publicación: 11 de marzo de 2017 
The field study was conducted in Oxapampa, Pasco, Peru to clinically diagnose bovine enzootic vesical hematuria (BEVH) by the urinalysis test, in conjunction with clinical and semiological evaluation of the animals, and the assessment of the farm. Urinary samples were collected by external manual stimulation of the perivulvar and perineal area in 211 female cattle, older than 6 months of age, and naturally exposed to consumption of braken fern - Pteridium aquilinum $(\mathrm{Pa})$. Samples were analyzed using reagent strips (RS), physicochemical characteristics (color, turbidity, specific weight and $\mathrm{pH}$ ), complementary chemical tests (protein, glucose and bilirrubin), and microscopic evaluation of the sediment (EMS). EMS is a confirmatory test for BEVH and highly relevant for subclinical cases (microhematuria). EMS results varied in intensity and therefore, a new classification based on the number of red blood cells per microscopic field was proposed. The relationship between EMS and RS, physicochemical test and complementary chemical tests were evaluated. A26.1\% prevalence of BEHV was found in the area. The statistical analysis showed that age and the interval to the last parturition can be considered as predisposing factors for $\mathrm{BEVH}$.

Key words: Bovine Enzootic Vesical Hematuria; urinalysis; EMS; RS; Pteridium aquilinum

\section{INTRODUCCIÓN}

La hematuria vesical enzoótica bovina (HVEB) es un proceso no infeccioso, tóxico, de curso crónico, caracterizado por una hematuria persistente, que varía de microhematuria a macrohematuria en periodos variables de tiempo, asociada con anemia y neoplasias del tracto urinario inferior (Jubb et al., 1993; Rebhun et al., 1995; McGavin et al., 2001). La macrohematuria es un signo que se asocia a problemas urológicos de importancia en más del $80 \%$ de los casos (Sutton, 1990) y a tumores vesicales en un $85 \%$ (Grossfeld y Carroll, 1998). La macrohematuria es la manifestación clínica más frecuente y constituye el primer signo en el $75 \%$ de los pacientes con neoplasias en la vejiga (Murphy et al., 1996).

La HVEB es ocasionada por el consumo prolongado del helecho común, Pteridium aquilinum (Pa), considerado una de las cinco malezas de mayor importancia en el mundo y una de las plantas tóxicas con mayor impacto en varios países (Alonso-Amelot,
1999; Marçal et al., 2001; Radostits et al., 2002). Este helecho se desarrolla en suelos ácidos y pobres en nutrientes ubicados entre 600 y 2000 msnm (Sánchez et al., 2006). Pa posee una molécula tóxica denominada dienona, responsable de las alteraciones genéticas que favorecen la formación de tejido disfuncional y canceroso en la vejiga urinaria del bovino (Alonso-Amelot, 1999). En salud pública, la presencia del ptaquilósido se asocia a la leche de vacas afectadas con el cáncer gástrico humano (Alonso-Amelot y Avendaño, 2002).

Gonzáles et al. (2004) hicieron la primera descripción en el Perú de las lesiones anatomo-histopatológicas de las neoplasias de vejigas de bovinos en Oxapampa. Posteriormente, a través de un estudio inmunohistoquímico, se determinó el origen blastodérmico de las neoplasias, demostrando la agresividad de las lesiones en los casos de HVEB (Montes, 2007). Estos estudios dejaron en evidencia la necesidad de contar con una técnica de diagnóstico clínico rápido, que permita identificar casos de HVEB desde los estadios subclínicos (microhematuria). 
Asimismo, los cambios frecuentes reportados en el examen de orina, como la presencia de eritrocitos y leucocitos, así como proteinuria y microhematuria (Peña et al., 2000), marcan un patrón importante para direccionar el diagnóstico clínico de la HVEB.

El análisis de orina es la prueba más importante para evaluar la enfermedad de vías urinarias (Merck, 2000; Bernard, 2005). Incluye pruebas físicas y químicas (evaluación de color, turbidez, peso específico y $\mathrm{pH}$ ), así como la evaluación microscópica del sedimento (EMS), la cual permite determinar la presencia clínica de hematuria (Campuzano y Gómez, 2007). Como parte del urianálisis también se incluye el método químico, que permite identificar proteinuria, aumento de nitrógeno ureico y baja gravedad específica, empleando tiras reactivas (TR), las que tienen alto grado de validez y seguridad para el diagnóstico de HVEB (Sánchez et al., 2006).

El objetivo del estudio fue diagnosticar y describir clínicamente la HVEB a través de un método validado para patologías del aparato urinario como el urianálisis, compuesto por una prueba tamiz por medio de TR, pruebas físico-químicas (color, turbidez, peso específico, $\mathrm{pH}$ ), pruebas químicas complementarias (glucosa, proteína, bilirrubina), y la EMS, que pueda ser utilizada como prueba clínica confirmatoria de hematuria y relevante para los casos subclínicos (microhematuria) de la HVEB.

\section{Materiales y Métodos}

\section{Población Animal}

El estudio se llevó a cabo en 2007 en bovinos hembras mayores de seis meses de edad, provenientes de centros de producción de leche y carne de crianza extensiva y de varias zonas de la provincia de Oxapampa (Pasco, Perú), ubicada a $1814 \mathrm{msnm}$. El tamaño de muestra fue de 211 animales, de- terminado a través de una prevalencia referencial de 16.34\% (Sánchez et al., 2006), un nivel de confianza del $95 \%$ y un error máximo admisible de 5\%.

Los animales fueron sometidos a una evaluación clínica y semiológica. Se registró la localidad, orientación productiva (leche, carne), edad (d 2,>2 a 4,>4), grupo racial (Bos taurus, Bos indicus, cruzado), número de partos $(0,1,2>3)$, condición corporal (escala de 1 a 5), fecha del último parto y tratamientos efectuados para descartar la presencia de otras posibles patologías. Las fincas seleccionadas eran manejadas bajo un sistema de producción de tipo extensivo con pastizales a base de gramíneas. Los pastizales estaban invadidos por frondes de Pteridium aquilinum. No se tuvo en consideración el tamaño de la finca ni el número de potreros.

\section{Muestras de Orina}

Las muestras de orina se obtuvieron mediante masaje manual perivulvar y perineal, previa limpieza de la zona. Se recolectó la fracción media de orina en frascos de plástico. Se evaluó la orina con las tiras reactivas SD UroColor ${ }^{\mathrm{TM}} 11$ para determinar sangre, urobilinógeno, proteínas, nitritos, cetonas, ácido ascórbico, bilirrubina, glucosa, $\mathrm{pH}$, gravedad específica y leucocitos. Se describieron las características macroscópicas de la orina color (amarillo, rojizo, marrón, entre otros) y nivel de turbidez; asimismo, se registró el peso específico utilizando un refractómetro y el $\mathrm{pH}$ mediante un pHmetro digital portátil, comparando los resultados obtenidos con los valores normales de acuerdo a la literatura (Benjamín, 1991).

Además, se determinaron los niveles de glucosa utilizando el reactivo de Benedict y la bilirrubina con el ácido nítrico y lugol (Benjamín, 1991). En el sedimento urinario se realizó el conteo directo de eritrocitos por campo microscópico, considerándose como muestra positiva la presencia de cinco a más eritrocitos por campo (Merck, 2000; Núñez et al., 2007). Esta observación permite con- 
Cuadro 1. Diagnóstico comparativo de Hematuria Vesical Enzoótica Bovina con la prueba de tiras reactivas y la evaluación microscópica del sedimento en bovinos de Oxapampa, Pasco

\begin{tabular}{lcccc}
\hline \multirow{2}{*}{ Diagnóstico } & & \multicolumn{2}{c}{$\begin{array}{c}\text { Tira Reactiva } \\
\text { (sangre) }\end{array}$} & \multirow{2}{*}{ Total } \\
\cline { 3 - 4 } & & Negativo & Positivo & \\
\cline { 3 - 4 } Sedimento & Negativo & 152 & 4 & 156 \\
& Positivo & 31 & 24 & 55 \\
\hline Total & & 183 & 28 & 211 \\
\hline
\end{tabular}

firmar los cuadros de hematuria en forma precisa desde sus inicios.

Los resultados de la EMS fueron expresados de acuerdo al número de eritrocitos observados por campo: HVEB Grado I = 5100 eritrocitos/campo; HVEB Grado II $=101$ 1000 eritrocitos/campo; HVEB Grado III = 1001- campo cubierto.

Los datos se analizaron mediante las pruebas de Chi cuadrado, Kappa, regresión logística y «t» Student, empleando el software de análisis de datos STATA 10.

\section{Resultados}

El 26.1\% (55/211) de las muestras de orina fueron positivas a hematuria detectadas por la EMS, mientras que solamente el $13.3 \%(28 / 211)$ de las muestras fueron positivas con las TR (Cuadro 1).

En el examen de los sedimentos se observó que 26 (47.3\%) de los 55 casos positivos fueron de grado I o leve, $12(21.8 \%)$ de grado II o moderado y 17 (30.9\%) de grado III o severo.

En la evaluación físico-química del urianálisis, al comparar los resultados del color y grado de turbidez versus el diagnóstico por la EMS, se encontraron diferencias significativas $(\mathrm{p}<0.05)$; observándose orinas aparentemente claras que podrían estar presentando fases severas de la enfermedad por la hematuria observada. Asimismo, en las pruebas químicas complementarias del urianálisis, el nivel de proteína en orina generó un valor significativo $(\mathrm{p}<0.05)$ asociado al diagnóstico de la HVEB mediante la EMS, mas no así el valor de glucosa.

En el $26.1 \%$ de prevalencia estimada mediante la EMS, la mayor cantidad de animales positivos estuvo en el grupo mayor de 4 años de edad (34.8\%) y en los animales cruzados (37.1\%); no encontrándose diferencias estadísticas entre el tipo de ganado o la procedencia del mismo con la presentación de HVEB (Cuadro 2). Las variables que tuvieron significancia estadística como factores predisponentes a la HVEB fueron la edad, grupo racial e intervalo al último parto $(\mathrm{p}<0.05)$ (Cuadro 3).

\section{Discusión}

La aplicación del urianálisis en el diagnóstico de la HVEB se concentra en el diagnóstico del principal signo clínico, la hematuria. Sin embargo, es un signo inespecífico que puede ser inducido por varios factores y causado por diferentes patologías. Como 
Cuadro 2. Prevalencia de Hematuria Vesical Enzoótica Bovina determinada por la evaluación microscópica del sedimento según el grupo etario, grupo racial, tipo de ganado y zona geográfica, en bovinos de Oxapampa, Pasco

\begin{tabular}{llccc}
\hline \multirow{2}{*}{ Variable } & & Total & Positivos & $\% \pm$ IC $^{1}$ \\
\cline { 4 - 5 } & & 34 & 4 & 11.8 \\
Edad (años) & $\leq 2$ & 88 & 20 & 22.7 \\
& $>2$ a 4 & 89 & 31 & 34.8 \\
Grupo racial & $>4$ & 18 & 6 & 33.3 \\
& Bos indicus & 104 & 16 & 15.4 \\
& Bos taurus & 89 & 33 & 37.1 \\
Tipo de ganado & Leche & 60 & 18 & 30.0 \\
& Carne & 151 & 37 & 24.5 \\
Zona & Cantarizú & 59 & 15 & 25.4 \\
& Chontabamba & 47 & 1 & 2.1 \\
& Oxapampa & 98 & 38 & 38.8 \\
& Tambo María & 7 & 1 & 14.3 \\
\hline Total & & 211 & 55 & $26.1 \pm 6.0$ \\
\hline
\end{tabular}

${ }^{1}$ Intervalo de confianza del 95\%

Cuadro 3. Evaluación de las variables edad, grupo racial, procedencia, tipo de ganado, número de partos, intervalo al último parto y condición corporal como factores predisponentes de Hematuria Vesical Enzoótica Bovina diagnosticada por la evaluación microscópica del sedimento en bovinos de Oxapampa, Pasco

\begin{tabular}{lccc}
\hline \multirow{2}{*}{ Variable } & $\mathrm{P}>|\mathrm{z}|$ & \multicolumn{2}{c}{ Intervalo de confianza 95\% } \\
\cline { 3 - 4 } & & Inferior & Superior \\
\hline Edad & 0.007 & 1.20 & 3.09 \\
Grupo racial & 0.036 & 1.04 & 2.95 \\
Procedencia & 0.054 & 0.99 & 2.02 \\
Tipo de ganado & 0.413 & 0.39 & 1.47 \\
Número de partos & 0.068 & 0.98 & 1.54 \\
Intervalo al último parto & 0.038 & 1.02 & 2.43 \\
Condición corporal & 0.170 & 0.49 & 1.13 \\
\hline
\end{tabular}


parte del uriánalisis se trabajó la EMS, el cual posee un alto porcentaje de sensibilidad para identificar casos de hematuria y, además, permite la cuantificación de eritrocitos para determinar la intensidad de la hematuria. Por ello, la EMS viene a ser la prueba de referencia para identificar casos de HVEB, lo cual marca una diferencia frente al método químico de las Tiras Reactivas (TR), el cual cumple un papel como prueba tamiz para identificar sangre en orina, teniendo la única ventaja sobre la EMS que permite evaluar también otros parámetros.

Al comparar los resultados de ambas pruebas, no se encontró un nivel de concordancia significativo entre ambas (Cuadro 1). En el caso de la EMS, es bastante improbable obtener falsos positivos a hematuria si la toma de muestras de orina se ha realizado de forma adecuada. La presencia de eritrocitos en un nivel mayor a cinco eritrocitos por campo en el sedimento indica hematuria (Merck, 2000; Núñez y Bouda, 2007). Por el contrario, la prueba con la TR requiere de un mayor número de eritrocitos para que ocurra una reacción química y obtener un cambio en el color de la tira. Esta reacción puede afectarse por varios factores y esto se observó en los cuatro animales positivos con la TR que fueron negativos a la EMS. Esto difiere de lo planteado por Kaneko et al. (1997) y Falbo et al. (2005), quienes hablan de una alta sensibilidad de la TR para la detección de eritrocitos y que es corroborado por el estudio de Sánchez et al. (2006) donde se halló niveles de verosimilitud entre ambas pruebas. Esta amplia diferencia entre la TR y la EMS en el presente estudio podría deberse a que la TR empleada fue de un laboratorio fabricante diferente al empleado en otros estudios; además, es una tira para orina humana que no ha sido diseñada para analizar orinas de un $\mathrm{pH}$ alcalino como la de los rumiantes.

La HVEB ha sido clínicamente clasificada como microhematúrica, refiriéndose a la forma inicial e imperceptible del proceso (forma subclínica) y macrohematúrica, cuando ya es evidente el cambio de coloración de la orina, de café a rojizo (forma clínica) (Jubb et al., 1993). En la práctica, esto dificulta un programa de control ya que conduce al error de considerar únicamente como animales positivos a los casos clínicos, cuando la hematuria es evidente. Esta clasificación de tipo cualitativa posee un componente de subjetividad muy alto debido a que no especifica el momento exacto, cuando la enfermedad pasa de la fase subclínica a clínica, que ocurre de forma progresiva en periodos variables de tiempo. Ante ello, se propuso una clasificación clínica de la HVEB de tipo cuantitativo, basado en la cantidad de eritrocitos por campo que se encuentran a la EMS, considerando como nivel basal o normal el valor de 0-4 eritrocitos por campo, según Merck (2000) y Núñez y Bouda (2007). No obstante, Chew y Di Bartola (1998), afirman que una muestra de orina sería negativa a hematuria con valores menores de 5 a 8 eritrocitos por campo, dependiendo de la técnica utilizada para la recolección.

Esta necesidad se confirmó dada la dispersión de los resultados considerados positivos a HVEB. Por ello, para un mejor análisis de los resultados se planteó una clasificación de los casos positivos a HVEB con tres niveles de enfermedad. Esta clasificación es únicamente de alcance clínico y no pretende competir con una necropsia o histopatología, sustentando su utilidad en el ordenamiento de los resultados de un monitoreo de HVEB en una finca para un mejor análisis y discusión de los mismos.

Se halló diferencia significativa cuando se comparó la relación del grado de enfermedad con el color de la orina. Es así que muestras de orinas de grado I y II no siempre son ligeramente rojizas o rojizas, y que una muestra de grado III podría aun verse amarilla como una orina normal, pudiendo estar relacionado a la integridad o lisis celular. También se halló diferencia significativa al comparar los grados de HVEB con el grado de turbidez de la orina, observándose que una orina grado I es preferentemente clara y una orina grado III es ligeramente turbia o 
turbia por el gran contenido de células rojas sanguíneas que posee.

No se encontró una asociación estadística entre los niveles de $\mathrm{pH}$ y peso específico con el diagnóstico de la enfermedad. Esto indica que la variación del $\mathrm{pH}$ en los casos positivos de HVEB no muestra un patrón constante, por lo que no resulta ser un indicador de HVEB. Respecto al peso específico, no se observó aumento en partículas químicas (iones) en la orina, lo cual se esperaría encontrar en alteraciones de la función renal (Chew y Di Bartola, 1998).

$\mathrm{Al}$ analizar la relación del diagnóstico de HVEB a través de la EMS en comparación a las pruebas químicas glucosa y proteína mediante regresión logística se encontró que únicamente el valor de proteína muestra significancia $(\mathrm{p}<0.05)$, lo cual se esperaría debido que a un mayor contenido de células en la orina se tiene por consecuencia proteinuria, como también lo reafirman Kaneko et al. (1997) y Falbo et al. (2005).

El uso de la EMS permitió determinar una prevalencia en Oxapampa de $26.1 \% \pm$ 0.06 (Cuadro 2), nivel alto que confirma a esta región como zona de riesgo para la HVEB. Este valor es muy similar al $24 \%$ reportado por Peña et al. (2000) en España trabajando con TR, y del 16\% observado por Sánchez et al. (2004) en Zulia, Venezuela, en condiciones medioambientales similares a Oxapampa, empleando las mismas técnicas diagnósticas.

La edad, grupo racial e intervalo al último parto fueron las únicas variables que mostraron significancia estadística, para ser considerados como factores predisponentes a la HVEB (Cuadro 3). Los animales con mayor edad tienen un mayor tiempo de exposición al consumo y, por consiguiente, mayor opción a desarrollar la enfermedad (Radostits et al., 2002). Asimismo, el intervalo al último parto es una variable considerada en el trabajo, debido a que en la práctica se observan casos de hematuria en animales recién paridos, lo cual podría estar sustentado en variaciones del sistema inmunitario en el periparto. No hubo relación entre el diagnóstico de HVEB con el número de partos, ya que en condiciones de crianza en selva alta una vaca puede tener 4 años y recién tener un parto; es decir, menor número de partos no necesariamente indica menor predisposición a la enfermedad. Respecto a la variable grupo racial, la literatura revisada no hace referencia a este como factor condicionante de la enfermedad, y al no tener animales con registros que permitan garantizar la pureza genética se sugiere considerar este dato únicamente como referencial.

\section{Conclusiones}

- El urianálisis es una herramienta efectiva para el diagnóstico de la HVEB, siendo considerada la evaluación microscópica de sedimento (EMS) como una prueba de referencia para diagnosticar animales positivos a HVEB.

- La tira reactiva (TR) presentó un bajo nivel de concordancia con la prueba de EMS.

- La prevalencia de $26.1 \%$ reportada en el presente estudio demuestra la condición de zona endémica a la HVEB en los vacunos de la zona de Oxapampa, Perú.

- La edad del animal y el intervalo al último parto pueden considerarse como factores predisponentes de la HVEB.

\section{Literatura Citada}

1. Alonso-Amelot ME. 1999. Helecho macho, salud animal y salud humana. Rev Fac Agron LUZ 16: 528-541.

2. Alonso-Amelot ME, Avendaño M. 2002. Human carcinogenesis and bracken fern: a review of the evidence. Curr Med Chem 9(6): 675-686. 
3. Benjamín MM. 1991. Manual de patología clínica en veterinaria. $3^{\mathrm{a}}$ ed. México: Limusa. $421 \mathrm{p}$.

4. Bernard J. 2005. El laboratorio en el diagnóstico clínico. Vol 1.20a ed. España: Marban Libros. 184 p.

5. Campuzano M, Gómez A. 2007. E1 urianálisis: un gran aliado del médico. Rev Urol Colomb 16(1): 67-92.

6. Chew J, Di Bartola S. 1998. Interpretación del urianálisis canino y felino. Wilmington, USA: Gloyd Group. 79 p.

7. Falbo M, Faria dos Reis A, Balarin M, Bracarense A, Araújo J, Okano W, Sandinia I. 2005. Alterações hematológicas, bioquímicas, urinárias e histopatológicas na intoxicação natural em bovinos pela samambaia Pteridium aquilnum (L.) Kühn. Semina Ciênc Agra 26: 547-558.

8. Gonzáles C, Chavera A, Perales $R$, Falcón N, Evaristo R. 2004. Caracterización de las lesiones encontradas en bovinos con hematuria vesical enzoótica en la zona de Oxapampa, Pasco. Rev Inv Vet Perú 15: 25-36. doi: 10.15381/ rivep.v15il.1559

9. Grossfeld GD, Carroll PR. 1998. Evaluation of asymptomatic microscopic hematuria. Urol Clin North Am 25: 661-676.

10. Jubb KVF, Kennedy PC, Palmer N. 1993. Pathology of domestic animals. Vol 2. $4^{\text {th }}$ ed. USA: Acad Press. 747 p.

11. Kaneko JR, Harvey JW, Bruss ML. 1997. Clinical biochemistry of domestic animals. $5^{\text {th }}$ ed. USA: Acad Press. 294 p.

12. Marçal WS, Gaste L, Reichert Netto NC, Gargantini M, Fernandes RP, Monteiro AA. 2001. Ocorrência de intoxicação aguda em bovinos pela samambaia (Pteridium aquilinum, L. Kuhn) no norte do Paraná-Brasil. Semina Ciênc Agra 22: 139-144.

13. McGavin MD, Carlton WW, Zachary JF. 2001. Thomson's special veterinary pathology. $3^{\text {rd }}$ ed. St Louis: Mosby. 755 p.

14. Merck. 2000. El manual Merck de veterinaria. $5^{\mathrm{a}}$ ed. Barcelona: Océano. 2455 p.
15. Montes K. 2007. Estudio inmunohistoquímico de la HVEB. Tesis de Médico Veterinario. Lima: Facultad de Medicina Veterinaria, Univ Nacional Mayor de San Marcos. 65 p.

16. Morales G, Valero F, Gaona J. 2003. Hematuria microscópica. Urol Colomb 12(1):31-44.

17. Murphy GP, Lawrence W, Lenhard RE. 1996. Oncología clínica - Manual de la American Cancer Society. $2^{\mathrm{a}}$ ed. EEUU: Organización Panamericana de la Salud. $870 \mathrm{p}$.

18. Núñez L, Bouda J. 2007. Patología clínica veterinaria. México: UNAM. 345 p.

19. Peña LF, Rodríguez A, Blanco J, Pérez M, Gonzáles M, Sánchez M, et al. 2000. Hallazgos clínicos y anatomopatológicos de la hematuria enzoótica bovina en una explotación de Avileña-Negra Ibérica. En: VII Congreso Internacional de Medicina Bovina. Oviedo. España.

20. Radostits OM, Gay CC, Blood DC, Hinchcliff $K W$. 2002. Medicina veterinaria: tratado de las enfermedades del ganado bovino, ovino, porcino, caprino y equino. Vol 2. $9^{\mathrm{a}}$ ed. España: McGraw Hill-Interamericana. $2215 \mathrm{p}$

21. Rebhun WC, Guard C, Richards CM. 1995. Diseases of dairy cattle. Philadelphia, USA: Williams \& Wilkins. $530 \mathrm{p}$.

22. Sánchez AV, Arraga CM, García D, Pino D, Villarroel R, Boscán J. 2004. Observaciones clínico patológicas en vacas con hematuria enzoótica bovina. Rev Cient (Maracaibo) 14: 317-323.

23. Sánchez AV, Arraga CM, Villarroel R, Pino D, García D, Sánchez G. 2006. Validez, seguridad y coeficiente de verosimilitud de los métodos tiras reactivas para orina y examen microscópico del sedimento urinario en el diagnostico de hematuria enzoótica bovina. Rev Cient (Maracaibo) 16: 604-612.

24. Sutton J. 1990. Evaluation of hematuria in adults. JAMA 263: 2475-2480. 